\title{
Carbon nanotubes quench singlet oxygen generated by photosynthetic reaction centers
}

\author{
Peter Boldog${ }^{1}$, Kata Hajdu“, , Melinda Magyar ${ }^{1}$, Éva Hideg ${ }^{2}$, Klara Hernádi ${ }^{3}$, Endre Horváth ${ }^{4}$, \\ Arnaud Magrez ${ }^{4}$, Krisztina Nagy ${ }^{5}$, György Váró ${ }^{5}$, Laszlo Forró ${ }^{4}$, and Laszlo Nagy ${ }^{1}$ \\ ${ }^{1}$ Department of Medical Physics and Informatics, University of Szeged, Szeged, Hungary \\ ${ }^{2}$ Institute of Biology, University of Pécs, Pécs, Hungary \\ ${ }^{3}$ Department of Applied and Environmental Chemistry, University of Szeged, Szeged, Hungary \\ ${ }^{4}$ Institute of Physics of Condensed Matter Physics, Ecole Polytechnique Federale de Lausanne, 1015 Lausanne, Switzerland \\ ${ }^{5}$ Institute of Biophysics, BRC, Szeged, 6726 Szeged, Hungary
}

Received 15 April 2013, revised 22 July 2013, accepted 10 September 2013

Published online 21 October 2013

Keywords carbon nanotube, reaction center protein, singlet oxygen

*Corresponding author: e-mail hajdu.kata@gmail.com, Phone/Fax: +36 62 544121, Web: www2.sci.u-szeged.hu/ant/

Photosensitizers may convert molecular oxygen into reactive oxygen species (ROS) including, e.g., singlet oxygen $\left({ }^{1} \mathrm{O}_{2}\right)$, superoxide anion $\left(\mathrm{O}_{2}^{-\bullet}\right)$, and hydroxyl radicals $\left({ }^{\circ} \mathrm{OH}\right)$, chemicals with extremely high cyto- and potential genotoxicity. Photodynamic ROS reactions are determinative in medical photodynamic therapy (cancer treatment with externally added photosensitizers) and in reactions damaging the photosynthetic apparatus of plants (via internal pigments). The primary events of photosynthesis take place in the chlorophyll containing reaction center protein complex (RC), where the energy of light is converted into chemical potential. ${ }^{1} \mathrm{O}_{2}$ is formed by both bacterial bacteriochlorophylls and plant RC triplet chlorophylls in high light and if the quenching of ${ }^{1} \mathrm{O}_{2}$ is impaired. In plant physiology, reducing the formation of the ROS and thus lessening photooxidative membrane damage (including the RC protein) and increasing the efficiency of the photochemical energy conversion is of special interest. Carbon nanotubes, in artificial systems, are also known to react with singlet oxygen. To investigate the possibility of ${ }^{1} \mathrm{O}_{2}$ quenching by carbon nanotubes in a biological system, we studied the effect of carbon nanotubes on ${ }^{1} \mathrm{O}_{2}$ photogenerated by photosynthetic RCs purified from purple bacteria. 1,3-Diphenylisobenzofuran (DPBF), a dye responding to oxidation by ${ }^{1} \mathrm{O}_{2}$ with absorption decrease at $420 \mathrm{~nm}$ was used to measure ${ }^{1} \mathrm{O}_{2}$ concentrations. ${ }^{1} \mathrm{O}_{2}$ was produced either from a photosensitizer (methylene blue) or from triplet photosynthetic RCs and the antioxidant capacity of carbon nanotubes was assessed. Less ${ }^{1} \mathrm{O}_{2}$ was detected by DPBF in the presence of carbon nanotubes, suggesting that these are potential quenchers of this ROS.
1 Introduction The primary events of photosynthesis take place in reaction center proteins (RC), where the energy of light is converted into chemical potential. Under conditions when the RC photochemistry is oversaturated reactive oxygen species (ROS,) are formed with large probability [1-3].

There is a large interest to reduce the formation of the ROS components because they may reduce the efficiency of photochemical energy conversion. In addition, they react with the intracellular components resulting in their degradation (the RC itself as well) [3].
Different mechanisms are developed in nature in order to decrease the ROS concentration, including specific enzyme reactions (e.g., peroxidases, superoxide dismutase enzymes) and/or decaying the concentration of long lived excited species (e.g., energy transfer from chlorophyll triplets to carotenoids, Fig. 1).

One of the most common ways to generate singlet oxygen is photosensitization by specific dyes (rose bengal, rhodamine derivatives, methylene blue (MB), etc.), which are able to transfer the excitation energy to triplet oxygen [4]. Due to their extensive $\pi$-electron systems $\mathrm{sp}^{2}$ carbon 


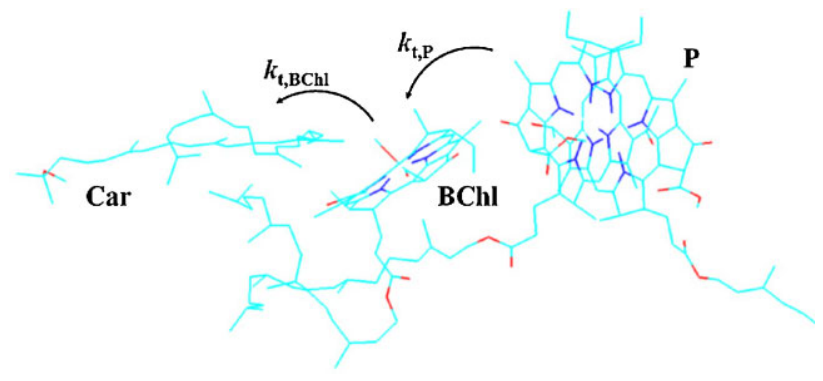

Figure 1 Schematic representation of triplet energy transfer from the excited primary electron donor (bacteriochlorophyll dimer, P) to the carotenoid (Car) in wild type Rhodobacter (Rb.) sphaeroides purple bacteria. BChl: bacteriochlorophyll monomer; $k_{\mathrm{t}, \mathrm{P}}$ and $k_{\mathrm{t}, \mathrm{BChl}}$ are rate constants of triplet energy transfer.

nanomaterials (like fullerenes, carbon nanotubes, nanohorns, and graphene) have unique dual properties. Besides fullerenes, which are well known as ${ }^{1} \mathrm{O}_{2}$ photosensitizers [5], ROS may also be generated by single walled carbon nanotubes (SWCNTs) in aqueous solution within the solar spectrum $(\lambda=300-410 \mathrm{~nm})$ irradiation under oxic conditions [7]. In addition, efficient energy transfer was found through $\pi-\pi$ stacking within the carbon nanotube complexes, e.g., with perylene derivatives by Ernst et al. [6].

On the other hand, single walled carbon nanotubes have been proved to be effective ${ }^{1} \mathrm{O}_{2}$ deactivators via either the energy transfer from ${ }^{1} \mathrm{O}_{2}$ to lower-energy excited states of SWNTs [7] or several chemical reaction pathways [8].

\section{Experimentals}

2.1 RC preparation RCs were prepared routinely from Rhodobacter sphaeroides R-26 purple bacteria. The cell membrane was solubilized by detergent (LDAO, $N, N$-dimethyldodecylamine- $N$-oxide, Fluka) and purified by ammonium sulfate precipitation, followed by anionexchange chromatography (DEAE Sephacel, Sigma) [9]. The fractions of OD280/OD800 smaller than 1.50 were used for our experiments.

2.2 Singlet oxygen detection In our work we used 1,3-diphenylisobenzofuran (DPBF), a dye that responds to oxidation by singlet oxygen with a decrease of its main absorption band $[10,11] .{ }^{1} \mathrm{O}_{2}$ was generated by red excitation of a sensitizer (MB) as a model system or photosynthetic reaction center in the absence or presence of carbon nanotubes. The reaction solution containing DBPF, methylene blue (MB), and/or RC were illuminated with red light $(\lambda>620 \mathrm{~nm})$ in a $0.5 \mathrm{~cm}$ cuvette and whole absorption spectra were measured in every 15 th seconds by a UNICAM UV-4 double-beam spectrophotometer.

\section{Results and discussions}

3.1 Quantifying ${ }^{1} \mathrm{O}_{2}$ in a model system The mixture of MB (sensitizer) and DPBF (reactant) was illuminated and the logarithm of the absorption at $420 \mathrm{~nm}$ as compared to the untreated sample was plotted as a

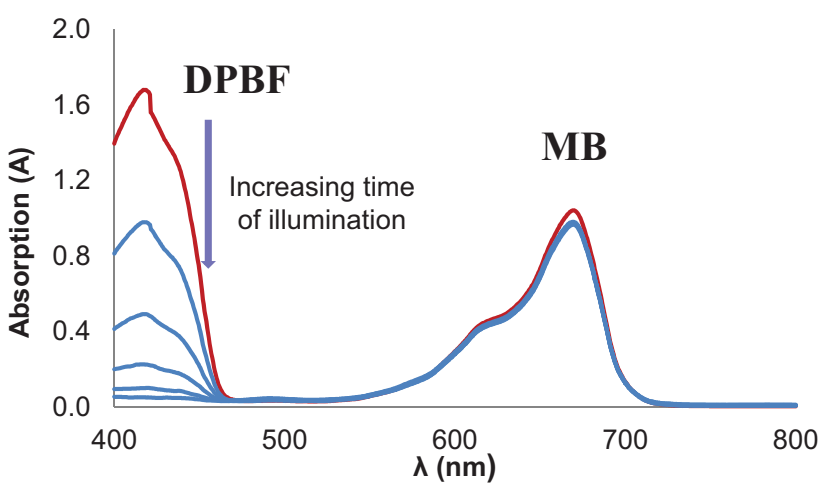

Figure 2 The absorption spectra of the DBF and MB solution after series of illumination. Arrow indicates increasing illumination times. The sample was measured in every 15 th minute.

function of the illumination time (dose-response curve, Figs. 2 and 3). Figures indicate that after illumination there is no change in the absorption of $\mathrm{MB}(665 \mathrm{~nm})$ while that of the DPBF decreases gradually.

\subsection{Study of the RC DPBF system}

3.2.1 DPBF binds to the RC Although DPBF is a well-known ${ }^{1} \mathrm{O}_{2}$ acceptor, it is not suitable for measurements in $\mathrm{H}_{2} \mathrm{O}$-rich media, because it is insoluble in water, and tends to form dimmers that are unreactive to ${ }^{1} \mathrm{O}_{2}$ [12]. Size exclusion chromatography proved that DPBF accumulated in the micelles containing $N, N$-dimethyldodecylamine- $N$ oxide (LDAO) and the RCs.

The mixture of RC and DPBF in TL (10 mM TRIS, $100 \mathrm{mM} \mathrm{NaCl}, 0.03 \%$ LDAO, pH 8.0) buffer was run on a Sephadex G-50 column (size exclusion chromatography). Experiment shows the presence of RC and DPBF in the main fraction (fraction 3 in Fig. 4) after elution indicating that the dye is attached to the RC/LDAO micelles.

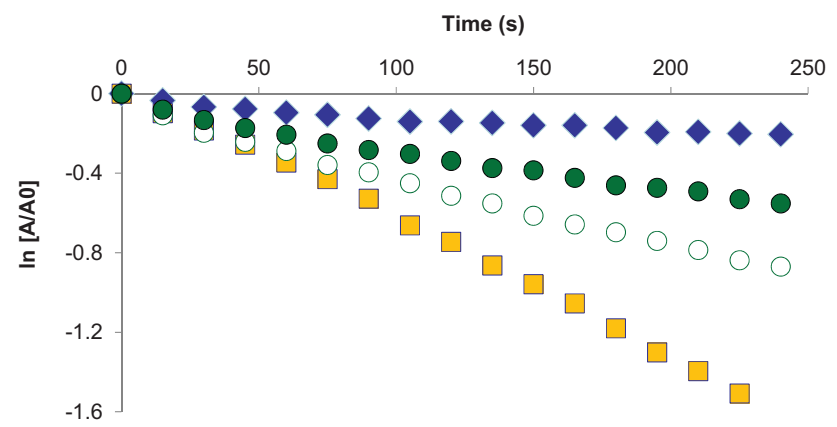

Figure 3 Normalized semi-logarithmic representation of the change in the DPBF absorption at $420 \mathrm{~nm}$ as a function of the illumination time. $A$ : absorption after given illumination time; $A_{0}$ : absorption without pre-illumination. Samples are: $\downarrow$-spontaneous degradation of DPBF; - DPBF, methylene blue, and carbon nanotubes $\left(14 \mathrm{mg} \mathrm{ml}^{-1}\right) ; \bigcirc-\mathrm{DPBF}$, methylene blue and carbon nanotubes $\left(7 \mathrm{mg} \mathrm{ml}^{-1}\right)$; - DPBF and methylene blue. Data represent results of single measurements. 


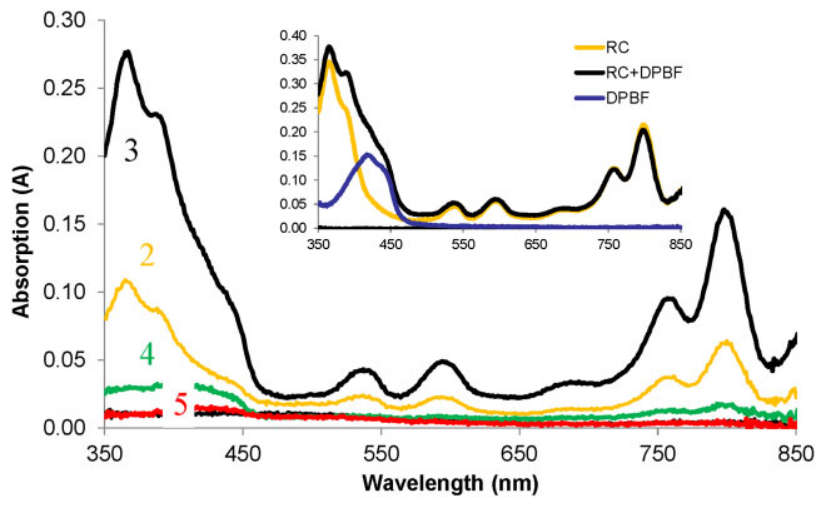

Figure 4 Elution fractions of the $\mathrm{RC}+\mathrm{DPBF}$ mixture. Insert shows the spectra of RC, DPBF and RC + DPBF solution in TL buffer.

\subsubsection{DPBF does not effect RC photochemistry If} we want to use DPBF in a RC mixture it is important to test whether it affects RC photochemistry. In response to single saturating flashes the RC performs charge separation that can be followed by measuring absorption change at $860 \mathrm{~nm}$. At this wavelength the redox state of the primary donor $\mathrm{P}$ can be followed. Figure 5 shows the absorption change of the RC solution after a single saturating flash in the presence and absence of DPBF. We found no difference in the absorption kinetics between samples in the absence and in the presence of DPBF.

\subsubsection{The role of carotenoid in ${ }^{1} \mathrm{O}_{2}$ formation by} RCs Photosystem II of plants do, wild purple bacteria do not generate ${ }^{1} \mathrm{O}_{2}$ upon illumination under aerobic conditions [2]. The diverse role of carotenoids in quenching ${ }^{1} \mathrm{O}_{2}$ in RC photochemistry is already demonstrated. The absence of any detectable amount of ${ }^{1} \mathrm{O}_{2}$ in RCs of wild type purple bacteria has been related with efficient Car quenching of triplets [3].

A dose-response curve of absorption change of DPBF induced by ${ }^{1} \mathrm{O}_{2}$ reaction in carotenoid containing (2.4.1.) and carotenoid-less (R-26) RCs indicates the difference between the two strains of RC (Fig. 6).

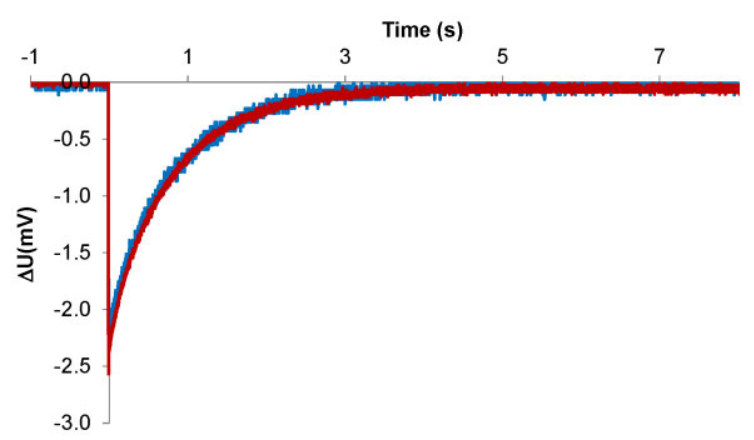

Figure 5 Absorption change of the RC solution after single saturating flash in the presence (red line) and absence (blue line) of DPBF. The measurement was done in TL buffer and the absorbance was measured at $860 \mathrm{~nm}$. Figure represents typical measurement.

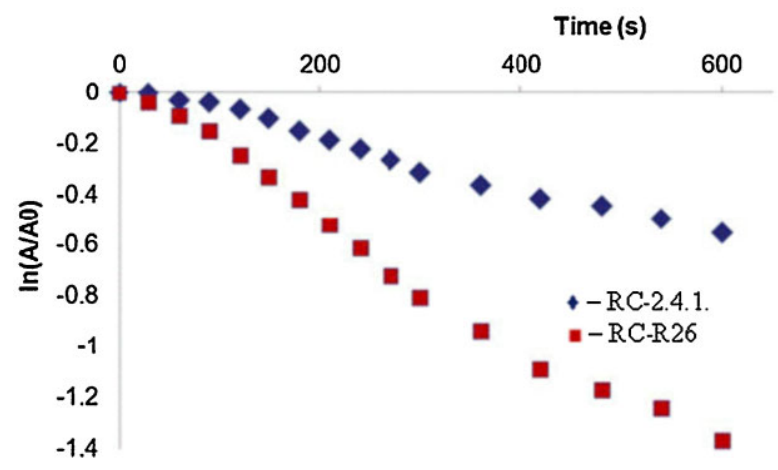

Figure 6 Normalized semi-logarithmic representation of the change in the DPBF absorption at $420 \mathrm{~nm}$ as a function of the illumination time of carotenoid containing (2.4.1.) and carotenoid less (R-26) RCs as indicated. A: absorption after given illumination time; $A_{0}$ : absorption without pre-illumination. Data represent results of single measurements.

3.3 Effect of carbon nanotubes on ${ }^{1} \mathrm{O}_{2}$ quenching in $\mathrm{RC}$ solutions RCs were bound specifically to $-\mathrm{NH}_{2}$ and $-\mathrm{COOH}$ functionalized multi-walled carbon nanotubes (MWNTs), $-\mathrm{NH}_{2}$ functionalized SWNTs, and physically to non-functionalized SWNTs.

Atomic force microscopy (AFM) image (Fig. 7) shows that it was possible to bind the $\mathrm{RC}$ to the aminefunctionalized carbon nanotubes using the glutaraldehyde (GTA) and a monolayer RC coating was formed on the CNT [13]. The carotenoid-less R-26 RCs were used in these experiments, because the wild type 2.4.1. quenches the singlet oxygen inherently.

Absorption change of RCs after single saturating flash excitation was measured when bound to single and multiwalled carbon nanotubes (similar measurements that was represented by Fig. 5). Data were analyzed assuming the first-order kinetic rate equation $A(t)=A_{i} \exp \left(-k_{i} t\right)$, which

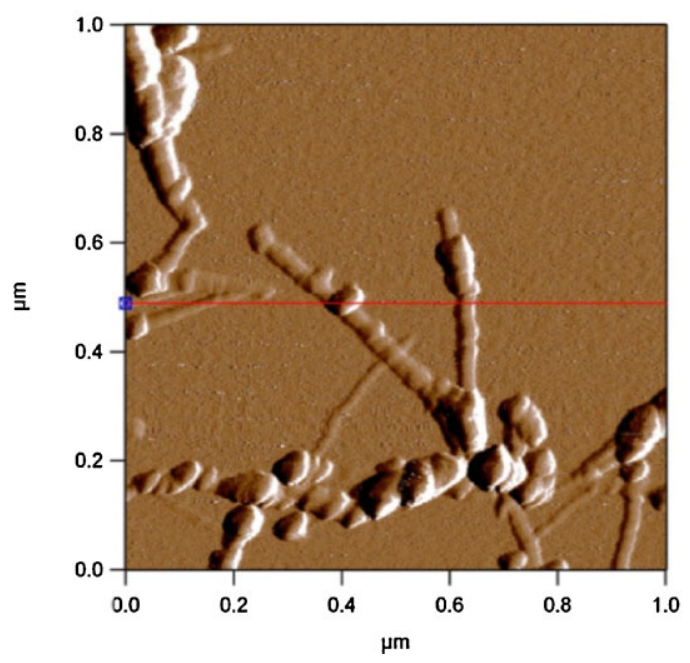

Figure 7 AFM image of SWNT/RC complex prepared by GTA activation. 
Table 1 Fitting parameters of the decay curves of absorption changes using different carrier matrices. The maximum error is $10.5 \%$. The $r^{2}$ of the fitting was 0.98 for the RCs in LDAO suspension and larger than 0.9 for the RC/NT complexes.

\begin{tabular}{lllll}
\hline & $A_{1}(\%)$ & $\tau_{1}(\mathrm{~ms})$ & $A_{2}(\%)$ & $\tau_{2}(\mathrm{~ms})$ \\
\hline RC/SWNT & 90 & 90 & 10 & 3100 \\
RC/MWNT & 88 & 90 & 12 & 2250 \\
RC in LDAO & 5 & 120 & 95 & 1200 \\
\hline
\end{tabular}

helps to detect multicomponent relaxations and their weight $\left(A_{i}\right)$ in the overall response. As the turnover of the RC should involve all reactions of the cycle, the slowest component will determine the overall turnover rate. The index $i=\mathrm{AP}$ (or $\mathrm{BP}$ ) corresponds to the $\mathrm{P}^{+} \mathrm{Q}_{\mathrm{A}}^{-} \mathrm{Q}_{\mathrm{B}} \rightarrow \mathrm{PQ}_{\mathrm{A}} \mathrm{Q}_{\mathrm{B}}\left(k_{\mathrm{AP}}\right)$ or to the $\mathrm{P}^{+} \mathrm{Q}_{\mathrm{A}} \mathrm{Q}_{\mathrm{B}}^{-} \rightarrow \mathrm{PQ}_{\mathrm{A}} \mathrm{Q}_{\mathrm{B}}\left(k_{\mathrm{BP}}\right)$ charge recombination $[3,13]$.

Using carbon nanotubes the ratio of the slow and fast component of the charge recombination after flash excitation is about 1 to 9 with SWNT and MWNT as well. The life time of the fast components remained unchanged, but that of the slow components increased compared to the one measured for clean RCs in LDAO suspension (Table 1). The contribution of the slow phase decreased considerably in the composites, probably, due to the low occupation of the secondary quinone site after the sample preparation.

As Fig. 8 shows, there is only small DPBF degradation when physical binding was applied. In our view at the first sample, this result can be due to the small LDAO concentration (in this sample the detergent concentration is well below the critical micellar concentration). Adding more detergent the effect was similar to the model system and the carbon nanotubes seemed to be quenchers of the arising ${ }^{1} \mathrm{O}_{2}$. When chemical binding was carried out large DPBF degradation was measured indicating an effective DPBF- ${ }^{1} \mathrm{O}_{2}$ reaction. There was no significant difference between the samples containing other types of the

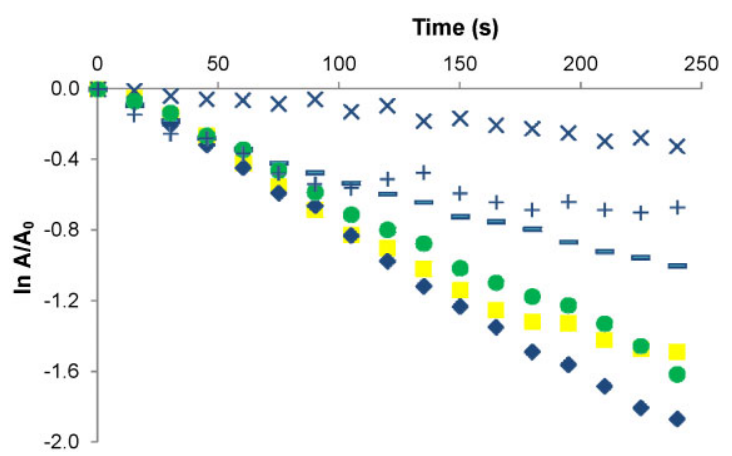

Figure 8 Dose-response curve of absorption change induced by DPBF- ${ }^{1} \mathrm{O}_{2}$ reaction in carotenoid-less $(\mathrm{R}-26) \mathrm{RCs}$ in the presence and absence of CNT. Samples: $(\times)-$ RC-R-26-physical binding, no detergent; (+) - RC-R-26-physical binding, with detergent; $(-)$ RC-R26 and DPBF; $(\mathbf{O})$ - SWCNT-NH $-\mathrm{RC} ;(\boldsymbol{\square})$ - MWCNT$\mathrm{NH}_{2}-\mathrm{RC} ;(\diamond)-\mathrm{MWCNT}-\mathrm{COOH}-\mathrm{RC}$. Data represent results of single measurements. carbon nanotubes, like single walled carbon nanotube or multi walled carbon nanotubes, and only a small difference was observed between the nanotubes owning different functional groups.

Results are in accordance with the finding presented in Fig. 4. DPBF is accumulated in the micellar system together with the RC and CNTs. The mean diffusion path $11.4 \pm 2 \mathrm{~nm}$ was reported by Kaplan and Trozzolo [14] or it can be estimated from the average life time $2-4 \mu \mathrm{s}[15,16]$ by the formula $x=\sqrt{4 D t}, 90-120 \mathrm{~nm}$ in aqueous solution. Here, $D$ is the diffusion coefficient of the ${ }^{1} \mathrm{O}_{2}$ and estimated to be $10^{-9} \mathrm{~m}^{2} \mathrm{~s}^{-1}$, and $t$ is the average life time. The $\mathrm{RC} / \mathrm{CNT}$ system falls in this range. The diameter of the RC, SWNT and MWNT are about 10, 2-4, and $10 \mathrm{~nm}$, respectively $[15,17]$.

No difference is seen between the deactivation processes in the presence of SWNT or MWNT. However, the influence of the $-\mathrm{NH}_{2}$ group is shown in agreement with earlier results that amines are good quenchers of ${ }^{1} \mathrm{O}_{2}$ [18]. Although data presented on Figs. 3, 6, and 8 represent results of single measurements these can be arranged in a consistent way and together with other measurements presented here a solid, reliable conclusion is confirmed.

4 Summary The equilibrium concentration of the ${ }^{1} \mathrm{O}_{2}$ is a result of the rate of the forward sensitization and the backward deactivation processes. Although, CNTs are known as ${ }^{1} \mathrm{O}_{2}$ sensitizers, under our conditions (short time far red light illumination) it is not probable that they play much role in generation of ${ }^{1} \mathrm{O}_{2}$, indeed, the main ${ }^{1} \mathrm{O}_{2}$ sensitizers are the carotenoid-less R-26 RCs.

The deactivation of ${ }^{1} \mathrm{O}_{2}$ in our system should be a matter of detailed further investigation. Here we present the first results in this system, but the phenomenon should be very complicated and worth to investigate. Carotenoids quench the triplet excited state of BChls in the RCs and/or react with the ${ }^{1} \mathrm{O}_{2}$ once it is formed. CNTs can be sensitized by ${ }^{1} \mathrm{O}_{2}$ directly depending the band structure (conductivity, chirality, thickness, functionalization, etc.) and/or react chemically through, typically, cycloaddition reactions. The detailed investigations are currently in progress.

Acknowledgements Work was supported by grants from Switzerland through the Swiss National Science Foundation (IZ73Z0_128037/1), the Swiss Contribution (SH/7/2/20), the Hungarian OTKA (K81180). The Project named "TÁMOP4.2.2/B-10/1-2010-0012-Creating the Center of Excellence at the University of Szeged" is supported by the European Union and co-financed by the European Social Fund.

\section{References}

[1] A. Telfer, S. M. Bishop, D. Philips, and J. Barber, J. Biol. Chem. 269, 13244-13253 (1994).

[2] J. B. Arellano, Y. A. Yousef, T. B. Melø, S. B. B. Mohamad, R. J. Cogdell, and K. R. Naqvi, J. Photochem. Photobiol. 87, 105-112 (2007).

[3] J. Tandori, É. Hideg, L. Nagy, P. Maróti, and I. Vass, Photosynth. Res. 70, 175-184 (2001). 
[4] F. Stracke, Ma. Heupel, and E. Thiel, J. Photochem. Photobiol. A 126, 51-58 (1999).

[5] M. K. Nissen, S. M. Wilson, and M. L. W. Thewalt, Phys. Rev. Lett. 69, 2423 (1992).

[6] F. Ernst, T. Heek, A. Setaro, R. Haag, and S. Reich, Adv. Funct. Mater. 22, 3921-3926 (2012).

[7] C.-Y. Chen and C. T. Jafvert, Carbon 49, 5099-5106 (2011).

[8] S. Lebedkin, I. Kareev, F. Hennrich, and M. M. Kappes, J. Phys. Chem. C 112, 16236-16239 (2008).

[9] J. Tandori, L. Nagy, A. Puskas, M. Droppa, G. Horvath, and P. Maroti, Photosynth. Res. 45, 135-146 (1995).

[10] M. A. Hamon, K. L. Stensaas, M. A. Sugar, K. C. Tumminello, and A. K. Allred, Chem. Phys. Lett. 447, 1-4 (2007).

[11] F. Amat-Guerri, E. Lempe, E. A. Lissi, F. J. Rodriguez, and F. R. Trull, J. Photochem. Photobiol. A 93, 49-56 (1996).
[12] B. A. Lindig, M. A. J. Rodgers, and P. Schaap, J. Am. Chem. Soc. 102, 5590-5593 (1980).

[13] M. Dorogi, Z. Bálint, Cs. Mikó, B. Vileno, M. Milas, K. Hernádi, L. Forró, Gy. Váró, and L. Nagy, J. Phys. Chem. B 110, 21473-21479 (2006).

[14] M. L. Kaplan and A. M. Trozzolo, in: Singlet Oxygen, edited by H. H. Wasserman and R. W. Murray (Academic Press, New York, 1979), p. 575.

[15] P. B. Merkel and D. R. Kearns, J. Am. Chem. Soc. Commun. 94, 1029-1030 (1972).

[16] C. Schweitzer and R. Schmidt, Chem. Rev. 103, 1685-1757 (2003).

[17] K. Hajdu, T. Szabó, M. Magyar, G. Bencsik, Z. Németh, K. Nagy, A. Magrez, L. Forró, Gy. Váró, K. Hernádi, and L. Nagy, Phys. Status Solidi B 248(11), 2700-2703 (2011).

[18] D. Bellus, Adv. Photochem. 11, 105-205 (1978). 